> Les protéines RAF (A-RAF, B-RAF et C-RAF) constituent une famille de kinases jouant un rôle déterminant dans des événements cellulaires aussi importants que la différenciation, la prolifération ou la régulation de la survie. Dans cette revue, nous faisons le point sur la régulation de la survie cellulaire par ces kinases. Les mécanismes en jeu sont particulièrement intéressants dans le domaine du cancer. Des études récentes portant sur la régulation de l'apoptose par les kinases RAF permettent d'envisager des approches thérapeutiques prometteuses en oncologie. <

\section{Régulation de la survie cellulaire par les kinases de la famille RAF}

Antoine Galmiche, Zakaria Ezzoukhry

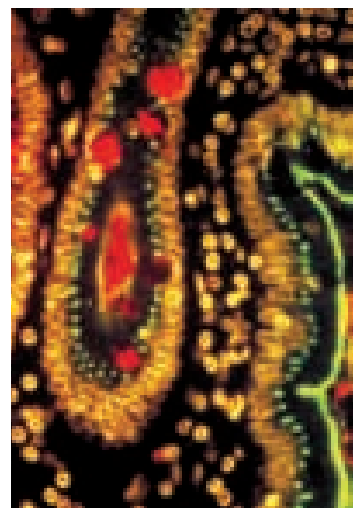

Laboratoire de biochimie, Inserm ERI12, CHU Nord Amiens, 1 , place Victor Pauchet, 80054 Amiens Cedex 1, France. galmiche.antoine@ chu-amiens. fr

\section{Structure et régulation des kinases RAF}

L'activation des kinases RAF est un des paramètres biochimiques les plus fréquemment altérés dans les tumeurs humaines [1]. Du fait de leur rôle oncogénique, les kinases de la famille RAF $^{1}$ ont d'emblée attiré l'attention des biologistes intéressés par la transduction du signal et la physiologie des cellules cancéreuses.

Chez les mammifères, la famille des kinases RAF est constituée de trois membres: A-RAF, B-RAF et C-RAF (anciennement Raf-1) [1]. Ces trois isoformes sont codées par des gènes distincts mais présentent d'importantes homologies de séquence (Figure l). Elles partagent une organisation commune avec un domaine carboxy-terminal qui porte l'activité sérine/thréonine kinase, alors que le domaine amino-terminal est régulateur et possède la particularité essentielle d'interagir avec les protéines RAS activées. Le domaine amino-terminal concentre l'essentiel des différences de séquence entre les kinases RAF.

Chez les vertébrés, les kinases RAF sont généralement exprimées dans tous les tissus, mais à des niveaux différents [1]. Les isoformes de RAF diffèrent entre elles en termes d'activité kinase. Toutes trois sont capables d'activer une unique cible, MEKl/2 (voir Glossaire), mais présentent d'importantes différences d'activité vis-à-vis de ce substrat [2-6]. Globalement, B-RAF est

${ }^{1}$ Voir le glossaire. la kinase la plus active et constitue probablement le principal activateur physiologique de la cascade MEKERK. La distribution subcellulaire des isoformes est également différente, notamment au niveau des membranes cellulaires. Alors que C-RAF interagit avec la mitochondrie, A-RAF est présent au niveau endosomal, et B-RAF est cytosolique [7]. L'ensemble de ces particularités suggère que, au-delà de la capacité partagée à activer MEK, chaque isoforme est impliquée dans des régulations cellulaires spécifiques.

L'interaction des GTPases RAS avec les kinases RAF est un événement critique pour leur activation. Suivant un modèle généralement accepté, cette interaction active directement les kinases RAF en prévenant une interaction inhibitrice entre les domaines amino-terminal régulateur et carboxy-terminal kinase $[8,9]$. En plus de leur effet activateur direct, les petites protéines $G$ de la famille RAS favorisent le recrutement des kinases RAF au niveau de structures membranaires spécialisées consistant en un regroupement local des isoformes de RAS entre elles [10]. À ce niveau, la présence de protéines d'échaffaudage comme KSR (kinase suppressor of Ras) et l'activité moindre des phosphatases facilitent la signalisation par les kinases $\operatorname{RAF}[10,11]$.

En plus de l'interaction avec RAS, les phosphorylations/ déphosphorylations jouent un rôle régulateur essentiel pour l'activité des RAF. Une région concentre plusieurs sites de phosphorylations stimulant directement l'activité des RAF kinases : il s'agit de la région N (nega- 


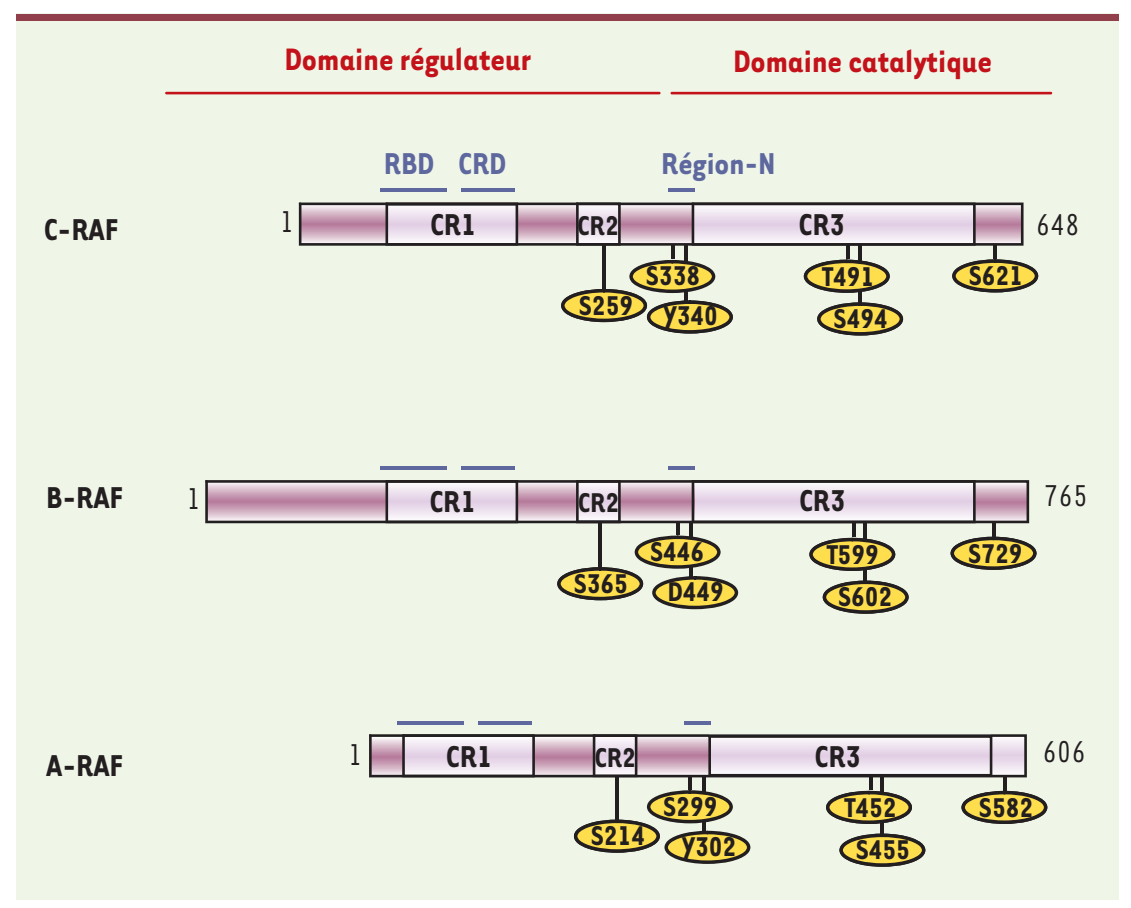

Figure 1. Structure des kinases RAF. Les isoformes de RAF (A-RAF, B-RAF, C-RAF) partagent trois régions conservées (les conserved regions $C R 1, C R 2$ et $C R 3$ ). Le CR1 consiste en un domaine d'interaction avec RAS (Ras binding domain, RBD) suivi par un domaine riche en Cys (cystein rich domain, CRD), le CR2 est une région riche en résidus sérine/thréonine, et le CR3 porte l'activité kinase. Globalement, les kinases RAF sont ainsi organisées en deux parties fonctionnelles: un domaine aminoterminal régulateur, incluant CR1 et CR2, et un domaine carboxy-terminal catalytique. La présence d'une extension amino-terminale constitue la principale différence entre les isoformes de RAF. On peut noter l'existence d'une courte région localisée devant le domaine kinase, qui régule l'activation, appelée la $\mathrm{N}$-région (pour marquer la présence de charges négatives à ce niveau). Plusieurs phosphorylations exerçant un rôle activateur sont concentrées dans la N-région, en particulier les résidus Ser 338 et Tyr 340 de C-RAF. Les phosphorylations activatrices directes de C-RAF portent également sur la boucle effectrice (Thr 491 et Ser 494). Le résidu Ser 621 constitue un résidu important pour le recrutement des protéines de la famille 14-3-3, car sa phosphorylation permet la formation de dimères entre C-RAF et B-RAF, ce qui constitue une étape importante de l'activation de ces kinases.

tively charged), en amont du domaine catalytique, qui joue un rôle régulateur important de l'activité kinase (Figure 1). Malgré la récente détermination de la structure cristalline du domaine catalytique de B-RAF [12], il n'existe que peu de données structurales pour expliquer l'effet régulateur direct des phosphorylations sur les RAF. De plus, certaines phosphorylations régulent indirectement l'activité kinase en changeant l'interaction des RAF avec d'autres protéines: en particulier, les protéines de la famille 14-3-3 qui reconnaissent des ligands phosphorylés et interagissent avec les kinases RAF. Elles permettent la formation de dimères entre les différentes kinases RAF, ce qui constitue une modalité importante de leur régulation [13]. À ce jour, les kinases MEKl/2, qui font partie du complexe d'activation de RAF, constituent l'unique substrat direct connu de ces kinases. Les kinases RAF ne régulent qu'indirectement leurs autres nombreux substrats, par l'intermédiaire de la cascade des kinases MEK-ERK.

\section{Effets anti-apoptotiques des kinases RAF}

Le rôle des kinases RAF dans la survie cellulaire a été clairement mis en évidence par les expériences d'invalidation des gènes codant pour CRaf et B-Raf chez la souris. L'invalidation de l'un ou l'autre de ces deux gènes cause une létalité embryonnaire. En l'absence de C-Raf, des niveaux d'apoptose importants sont observés dans plusieurs tissus, en particulier hépatique [14]. De la même façon, l'absence de B-Raf affecte la survie des cellules neuronales $[15,16]$. Ces travaux ont permis de mettre en évidence le rôle fondamental des kinases RAF dans le contrôle de la survie cellulaire. Dans les paragraphes qui suivent, nous faisons le point sur les mécanismes impliqués en présentant les cibles cytoplasmiques et nucléaires des voies RAF.

Substrats cytoplasmiques régulant la survie cellulaire La mitochondrie est l'organite essentiel dans la régulation de l'apoptose [17] et intègre l'essentiel des stimulus pro ou anti-apoptotiques. La perméabilisation de la membrane externe mitochondriale est une étape critique de l'exécution du programme apoptotique. Cette perméabilisation est sous le contrôle des protéines de la famille $\mathrm{BCL}^{1}{ }^{1}$ (B-cell lymphoma 2) [17]. Des travaux menés dès la fin des années 1990 ont mis en évidence la présence de C-RAF au niveau des mitochondries et l'effet anti-apoptotique de cette kinase (revue dans [18]). Depuis, de nombreuses études ont montré [18] que les kinases RAF régulent les protéines de la famille BCL2 [19]. Suivant les tissus et les types cellulaires étudiés, les kinases MEK-ERK activées en aval des RAF sont capables de neutraliser les protéines pro-apoptotiques comme BAD (BCLXL/BCL2-associated death promoter) ou BIM ( $\mathrm{BCl}-2$ interacting mediator of cell death) (Figure 2) et/ou d'activer les membres antiapoptotiques de cette famille de protéines comme BCL2 ou MCLI (myeloid leukemia cell differentiation pro- 
tein 1) [19]. Des mécanismes combinés de neutralisation directe et le contrôle du turn-over de ces protéines par le protéasome contribuent à inactiver les facteurs pro-apoptotiques. Ainsi, la voie RAF séquestre BAD dans le cytoplasme en créant un site d'interaction avec les protéines de la famille 14-3-3. La phosphorylation de BIM en aval de la voie RAF crée un site de reconnaissance pour l'ubiquitine ligase $\beta$-TRCP, favorisant de ce fait la dégradation de la protéine [19]. Au final, les kinases RAF préviennent donc l'activation de la machinerie apoptotique mitochondriale en contrôlant à la fois les niveaux d'expression et l'activité des protéines de la famille BCL2 par l'intermédiaire de la cascade MEK-ERK.

D'autres mécanismes cytoplasmiques sont également mis en jeu dans la régulation négative de l'apoptose. La phosphorylation du résidu thréonine 125 de la caspase-9 par les kinases ERK réduit l'activation de cette caspase [20]. De ce fait, les kinases RAF sont encore capables de prévenir l'apoptose en aval de la libération du cytochrome c. Finalement, d'autres mécanismes anti-apoptotiques ont été mis en évidence pour C-RAF. Ceux-ci présentent une particularité importante, à savoir leur indépendance vis-à-vis de l'activité kinase de C-RAF et, a fortiori, vis-à-vis de la cascade MEK-ERK. Ces effets anti-apoptotiques reposent sur la régulation négative par interaction physique de C-RAF avec plusieurs sérine/thréonine kinases qui exercent plus ou moins directement des effets pro-apoptotiques : les kinases MST2 (voir Glossaire) [21], ASK1 [22], ou ROK $\alpha$ [23] (Figure 2). Dans ce dernier cas, l'inhibition en trans de ROK $\alpha$ par C-RAF semble capable d'influencer la structure du cytosquelette d'actine et, indirectement, la signalisation par les récepteurs de mort cellulaire comme FAS [24].

\section{Régulation transcriptionnelle}

de la survie cellulaire par les kinases RAF

À côté des mécanismes cytoplasmiques mis en jeu par les kinases RAF, le contrôle mitochondrial de l'apoptose est également le fait d'une régulation transcriptionnelle. Dans plusieurs systèmes cellulaires, l'activation des kinases RAF induit une réduction des niveaux d'expression des ARNm codant pour BAD ou BIM en même temps qu'une augmentation de ceux codant pour BCL2, BCLXL (B-cell lymphoma-extra large) ou MCL1 [19]. Les kinases RAF régulent essentiellement la transcription de ces gènes par le biais de la cascade MEK-ERK [25]. Les kinases ERK activées peuvent en effet entrer dans le noyau des cellules pour réguler l'activité de nombreux facteurs de transcription, comme par exemple les facteurs de transcription ETS, FOS ou EGRl [26]. En plus de ces cibles bien identifiées, d'autres facteurs de transcription comme NF- $\kappa B$ jouent un rôle important dans la régulation de la survie cellulaire et la prévention de l'apoptose, suivant des mécanismes plus complexes [27]. Finalement, et de façon plus spéculative, les kinases RAF pourraient également réguler les marques épigénétiques. Un travail récent montre que l'activation de RAS module l'expression de la protéine pro-apoptotique FAS par le biais de mécanismes épigénétiques qui mettent en jeu les systèmes de modification et de remodelage de la chromatine comme les protéines Polycomb (BMI), les ADN méthylases, certaines protéines modifiant les histones (méthyl-transférases et histone désacétylases) [28]. Il sera important de déterminer dans quelle mesure les kinases RAF régulent la structure et l'accessibilité de la chromatine.

\section{Kinases RAF et survie de la cellule cancéreuse}

L'activation des kinases RAF constitue une des anomalies les plus fréquemment retrouvées dans les cancers humains $[1,41]$. Le plus souvent, les kinases RAF sont activées par le biais de dérèglements de l'activité des récepteurs à activité tyrosine kinase (typiquement le récepteur de I'EGF, epidermal growth factor) ou des petites protéines $G$ de la famille RAS (des mutations activatrices de ces GTPases sont fréquemment détectées dans les tumeurs du côlon ou du pancréas). Des mutations somatiques activatrices de B-RAF ont été plus

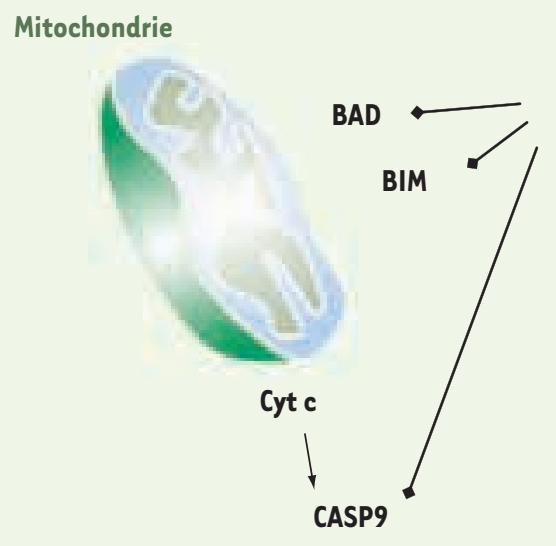

Mitochondrie
RSK

ERK

MEK

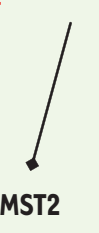

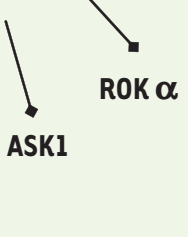

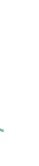

. 


\begin{tabular}{lcc}
\hline Inhibiteur (kinase ciblée) & Type de cancer & Référence \\
\hline Géfitinib (récepteur EGF) & Poumon & {$[36]$} \\
\hline Erlotinib (récepteur EGF) & Poumon & {$[37]$} \\
\hline U0126 (MEK) & Côlon & {$[38]$} \\
\hline Sorafénib (RAF) & Foie & [soumis] \\
\hline
\end{tabular}

Tableau I. Principales études mettant en évidence une synergie pro-apoptotique entre les inhibiteurs de la voie des kinases RAF et le composé BH3-mimétique ABT-737.

récemment mises en évidence dans les mélanomes et certaines tumeurs de la thyroïde et du côlon. Une mutation en particulier, B-RAF V600E, est de loin la plus souvent retrouvée. Cette mutation, localisée sur la boucle activatrice de B-RAF, maintient la kinase sous forme constitutivement active [12].

De façon intéressante, des travaux récents ont mis en évidence d'importantes différences physiologiques entre les cellules cancéreuses porteuses d'oncogènes RAF activés indirectement (activation de récepteurs ou mutations de RAS) et celles qui possèdent les mutations activatrices de $B-\operatorname{RAF}[29,30]$. Les cellules tumorales porteuses de l'oncogène B-RAF V600E présentent un phénomène d'addiction, c'est-à-dire de dépendance à des niveaux soutenus d'activation de la kinase MEK pour leur survie et leur croissance [29, 30]. Des données récentes indiquent que les altérations d'expression et d'activation des protéines régulant l'apoptose, et notamment les protéines de la famille $B C L 2$, jouent un rôle essentiel dans les phénomènes d'addiction des cellules cancéreuses vis-à-vis des voies de transduction oncogéniques $[19,31]$. Les cellules tumorales de mélanomes ou de cancers du côlon porteuses d'un oncogène $B-R A F$ mutant V600E sont capables d'inactiver BAD et BIM de façon dépendante de MEK et le blocage de MEK induit des niveaux accrus d'apoptose dans ces cellules [32-34]. Sur la base de ces nouvelles données, des travaux récents suggèrent l'efficacité d'un nouveau type d'association pharmacologique dans le traitement des cancers, avec d'une part des composés interférant avec les protéines $B C L 2$, et d'autre part les thérapies ciblées antagonisant la voie RAF [35]. Nous résumons dans le Tableau / les principales études rapportant un effet pro-apoptotique synergique entre le blocage de la voie RAF et le traitement par $A B T-737$, le principal inhibiteur à ce jour des protéines BCL2 [36-38]. Pour l'essentiel, les études évaluant ces synergies rapportent des résultats obtenus in vitro. II existe encore peu d'études qui reposent sur des modèles animaux de tumeurs [38].

\section{Conclusion}

Conformément à une conception émergente dans le domaine de la signalisation intracellulaire, les kinases RAF constituent des points de convergence dans la régulation de la survie cellulaire [39]. Une meilleure compréhension des mécanismes mis en jeu par ces kinases dans les cellules cancéreuses est requise pour comprendre leur rôle oncogénique. Ces recherches seront essentielles pour améliorer les traitements actuels des cancers, car provoquer la mort des cellules cancéreuses est une condition obligatoire pour obtenir une régression de ces tumeurs. Dans cette optique, des recherches prometteuses visent à identifier les mécanismes anti-apoptotiques qui pourraient être à l'origine de l'addiction des cellules cancéreuses aux kinases MEK, ou à identifier d'éventuels talons d'Achille de ces cellules, comme par exemple les anomalies du métabolisme [40]. Près de vingt-cinq ans après leur découverte, les kinases RAF constituent toujours un champ ouvert pour des recherches qui devraient être fertiles! $\diamond$

\section{GLOSSAIRE DES INTERMÉDIAIRES DE LA VOIE RAF}

ASK1 : apoptosis signal-regulating kinase 1

Cascade des protéines BCL2 : Les protéines de la famille BCL2 contrôlent les étapes de perméabilisation de la membrane externe mitochondriale permettant l'engagement de la cellule dans l'apoptose. Les protéines pro-apoptotiques comme BAX ou BAK sont des constituants centraux de la machinerie apoptotique. Les protéines anti-apoptotiques de la famille BCL2, comme BCL2, BCLXL ou MCL1, exercent un contrôle régulateur négatif sur $B A X$ et $B A K$. Ces protéines sont régulées par une sous-famille de molécules appartenant à la famille BCL2 et ne possèdant qu'un domaine d'homologie limité avec le reste de la famille, le domaine BH3, limité à environ 15 acides aminés. Certains membres de la famille des $B H 3$-only, comme BAD, neutralisent les protéines antiapoptotiques $B C L 2, B C L X L$, et agissent ainsi comme des sensibilisateurs à l'apoptose, alors que d'autres, comme BIM ou BID, sont aussi capables d'activer directement les protéines pro-apoptotiques comme BAX.

EGR1 : early growth response factor 1

ERK : extracellular signal-regulated kinase

ETS : ( $\varepsilon$-twenty six) famille de facteurs de transcription caractérisés par un domaine homologue de liaison à l'ADN d'environ 85 acides aminés.

FOS : facteur de transcription homologue de l'oncogène $v$-Fos du virus FBJ de l'ostéosarcome murin.

MAP-kinase : mitogen-activated protein kinase

MEK : MAP-kinase/ERK-kinase

MST2 : mammalian STE20-like kinase 2

RAF : kinases nommées par homologie avec l'oncogène $v$-raf. Les protéines RAF sont activées par les GTPases associées aux membranes de la famille RAS. Après activation, les protéines RAF peuvent activer par phosphorylation les kinases MEK1 et MEK2 qui activent à leur tour par phosphorylation les sérine/thréonine kinases ERKl et ERK2.

ROK : Rho kinase 


\section{SUMMARY}

\section{Regulation of cell survival by RAF kinases}

The RAF proteins ( $A-R A F, B-R A F$ et C-RAF) are a family of kinases that play a key role in the regulation of various aspects of cell physiology, among which cell proliferation, differentiation and survival. We review the litterature regarding their anti-apoptotic effects, and we summarize the current view regarding how alterations in these mechanisms contribute to human carcinogenesis. Finally, we discuss new therapeutic perspectives based on the manipulation of cell survival by the RAF kinase pathway. $\diamond$

\section{CONFLIT D'INTÉRÊTS}

Les auteurs déclarent n'avoir aucun conflit d'intérêts concernant les données publiées dans cet article.

\section{REMERCIEMENTS}

Nous remercions la Ligue contre le cancer - comité de la Somme-pour son soutien financier.

\section{RÉFÉRENCES}

1. Wellbrock C, Kasarides M, Marais R. The Raf proteins take centre stage. Nat Rev Mol Cell Biol $2004 ; 5: 875-85$.

2. Pritchard CA, Samuels ML, Bosch $\varepsilon$, McMahon M. Conditionally oncogenic forms of the A-Raf and B-Raf protein kinases display different biological and biochemical properties in NIH 3 T3 cells. Mol Cell Biol 1995; $15: 6430-42$.

3. Marais R, Light Y, Paterson HF, et al. Differential regulation of Raf-1, A-Raf, and B-Raf by oncogenic ras and tyrosine kinases. J Biol Chem $1997 ; 272: 4378-83$.

4. Papin C, Denouel-Galy A, Laugier D, et al. Modulation of kinase activity and oncogenic properties by alternative splicing reveals a novel regulatory mechanism for B-Raf. J Biol Chem 1998 ; $273: 24939-47$

5. Baljuls A, Mueller T, Drexler HC, et al. Unique $\mathrm{N}$-region determines low basal activity and limited inducibility of A-RAF kinase: the role of $\mathrm{N}$-region in the evolutionary divergence of RAF kinase function in vertebrates. J Biol Chem $2007 ; 282: 26575-90$.

6. Fischer A, Hekman M, Kuhlmann J, et al. B- and C-RAF display essential differences in their binding to Ras: the isotype-specific $N$ terminus of B-RAF facilitates Ras binding. J Biol Chem $2007 ; 282$ : 26503-16.

7. Galmiche A, Fueller J, Santel A, et al. Isoform-specific interaction of C-RAF with mitochondria. J Biol Chem 2008; 283 : 14857-66.

8. Cutler RE Jr, Stephens RM, Saracino MR, Morrison DK. Autoregulation of the Raf-l serine/threonine kinase. Proc Natl Acad Sci USA 1998 ; 95 : $9214-9$

9. Terai K, Matsuda M. Ras binding opens c-Raf to expose the docking site for mitogen-activated protein kinase kinase. EMBO Rep 2005; $6: 251-5$.

10. Harding AS, Hancock JF. Using plasma membrane nanoclusters to build better signaling circuits. Trends Cell Biol $2008 ; 18: 364-71$.

11. Chen C, Lewis RE, White MA. IMP modulates KSRl-dependent multivalent complex formation to specify ERK1/2 pathway activation and response thresholds. J Biol Chem 2008; 283 : 12789-96.

12. Wan PT, Garnett MJ, Roe SM, et al. Mechanism of activation of the RAF-ERK signaling pathway by oncogenic mutations of B-RAF. Cell $2004 ; 116: 855-67$.

13. Rajakulendran T, Sahmi M, Lefrançois $M$, et al. A dimerization-dependent mechanism drives RAF catalytic activation. Nature $2009 ; 461: 542-5$.

14. Mikula M, Schreiber M, Husak Z, et al. Embryonic lethality and fetal liver apoptosis in mice lacking the c-raf-1 gene. EMBO J 2001; $20: 1952-62$.

15. Wiese $S$, Pei G, Karch C, et al. Specific function of B-Raf in mediating survival of embryonic motoneurons and sensory neurons. Nat Neurosci $2001 ; 4$ : 137-42.

16. Galabova-Kovacs G, Matzen D, Piazzolla D, et al. Essential role of B-Raf in ERK activation during extraembryonic development. Proc Natl Acad Sci USA 2006 ; 103 : 1325-30.

17. Youle RJ, Strasser $A$. The BCL-2 protein family: opposing activities that mediate cell death. Nat Rev Mol Cell Biol $2008 ; 9:$ 47-59.

18. Galmiche A, Fueller J. RAF kinases and mitochondria. Biochim Biophys Acta 2007 ; 1773 : 1256-62.
19. Balmanno K, Cook SJ. Tumour cell survival signalling by the ERKI/2 pathway. Cell Death Differ 2009; 16 : 368-77.

20. Allan LA, Morrice N, Brady S, et al. Inhibition of caspase- 9 through phosphorylation at Thr 125 by ERK MAPK. Nat Cell Biol $2003 ; 5$ : 647-54

21. O'Neill $\varepsilon$, Rushworth L, Baccarini M, Kolch W. Role of the kinase MST2 in suppression of apoptosis by the proto-oncogene product Raf-1. Science $2004 ; 306: 2267-70$

22. Chen J, Fujii K, Zhang L, et al. Raf-1 promotes cell survival by antagonizing apoptosis signal-regulating kinase 1 through a MEK-ERK independent mechanism. Proc Natl Acad Sci USA $2001 ; 98$ : 7783-8.

23. Niault T, Sobczak I, Meissl K, et al. From autoinhibition to inhibition in trans: the Raf-1 regulatory domain inhibits Rok-alpha kinase activity. J Cell Biol $2009 ; 187: 335-42$.

24. Piazzolla D, Meissl K, Kucerova L, et al. Raf-1 sets the threshold of Fas sensitivity by modulating Rok-alpha signaling. J Cell Biol 2005; $171: 1013-22$.

25. Schulze A, Nicke B, Warne PH, et al. The transcriptional response to Raf activation is almost completely dependent on mitogen-activated protein kinase kinase activity and shows a major autocrine component. Mol Biol Cell $2004 ; 15: 3450-63$.

26. Whitmarsh AJ. Regulation of gene transcription by mitogen-activated protein kinase signaling pathways. Biochim Biophys Acta 2007 ; 1773 : 1285-98.

27. Liu J, Suresh Kumar KG, Yu D, et al. Oncogenic BRAF regulates beta-Trcp expression and NF-kappaB activity in human melanoma cells. Oncogene $2007 ; 26: 1954-8$

28. Gazin C, Wajapeyee N, Gobeil S, et al. An elaborate pathway required for Ras-mediated epigenetic silencing. Nature 2007 ; 449 : 1073-7.

29. Solit DB, Garraway LA, Pratilas CA, et al. BRAF mutation predicts sensitivity to MEK inhibition. Nature 2006 ; 439 : 358-62.

30. Pratilas CA, Taylor BS, Ye $Q$, et al. ${ }^{\mathrm{V} 600 \varepsilon B R A F}$ is associated with disabled feedback inhibition of RAF-MEK signaling and elevated transcriptional output of the pathway. Proc Natl Acad Sci USA 2009 ; 106 : 4519-24.

31. Letai AG. Diagnosing and exploiting cancer's addiction to blocks in apoptosis. Nat Rev Cancer 2008 ; 8 : 121-32.

32. Sheridan C, Brumatti G, Martin SJ. Oncogenic B-RafV600E inhibits apoptosis and promotes ERK-dependent inactivation of Bad and Bim. J Biol Chem $2008 ; 283: 22128-35$.

33. Boisvert-Adamo K, Aplin AE. Mutant B-RAF mediates resistance to anoikis via Bad and Bim. Oncogene 2008; 27 : 3301-12.

34. Wickenden JA, Jin $\mathrm{H}$, Johnson M, et al. Colorectal cancer cells with the BRAF(V600E) mutation are addicted to the $\varepsilon R K 1 / 2$ pathway for growth factor-independent survival and repression of BIM. Oncogene 2008; $27: 7150-61$.

35. Cragg MS, Harris C, Strasser A, Scott CL. Unleashing the power of inhibitors of oncogenic kinases through BH3 mimetics. Nat Rev Cancer 2009 ; 9 : 321-6.

36. Cragg MS, Kuroda J, Puthalakath $\mathrm{H}$, et al. Gefitinib-induced killing of NSCLC cell lines expressing mutant $\varepsilon$ GFR requires BIM and can be enhanced by $\mathrm{BH} 3$ mimetics. PLoS Med 2007 ; 4 : 1681-9.

37. Gong $Y$, Somwar R, Politi K, et al. Induction of BIM is essential for apoptosis triggered by EGFR kinase inhibitors in mutant EGFR-dependent lung adenocarcinomas. PLoS Med 2007 ; 4 : e294.

38. Cragg MS, Jansen ES, Cook M, et al. Treatment of B-RAF mutant human tumor cells with a MEK inhibitor requires Bim and is enhanced by a BH3 mimetic. J Clin Invest 2008; 118 : 3651-9.

39. Taniguchi CM, Emanuelli B, Kahn CR. Critical nodes in signalling pathways: insights into insulin action. Nat Rev Mol Cell Biol 2006; 7: 85-96.

40. Yagoda N, von Rechenberg M, Zaganjor $\varepsilon$, et al. RAS-RAF-MEK-dependent oxidative cell death involving voltage-dependent anion channels. Nature $2007 ; 447: 864-8$

41. Lavoie H, Therrien M. Mécanisme d'activation de l'oncogène BRAF. L'union fait la force. Med Sci (Paris) $2010 ; 26: 459-60$.

\section{TIRÉS À PART}

A. Galmiche 